This is an electronic reprint of the original article. This reprint may differ from the original in pagination and typographic detail.

Author(s): Morgan, Ian; Mansikkamäki, Akseli; Rouzières, Mathieu; Clérac, Rodolphe; Tuononen, Heikki

Title: $\quad$ Coexistence of long-range antiferromagnetic order and slow relaxation of the magnetization in the first lanthanide complex of a 1,2,4-benzotriazinyl radical

Year: $\quad 2017$

Version:

Please cite the original version:

Morgan, I., Mansikkamäki, A., Rouzières, M., Clérac, R., \& Tuononen, H. (2017). Coexistence of long-range antiferromagnetic order and slow relaxation of the magnetization in the first lanthanide complex of a 1,2,4-benzotriazinyl radical. Dalton Transactions, 46(38), 12790-12793. https://doi.org/10.1039/C7DT02766D

All material supplied via JYX is protected by copyright and other intellectual property rights, and duplication or sale of all or part of any of the repository collections is not permitted, except that material may be duplicated by you for your research use or educational purposes in electronic or print form. You must obtain permission for any other use. Electronic or print copies may not be offered, whether for sale or otherwise to anyone who is not an authorised user. 


\title{
Coexistence of long-range antiferromagnetic order and slow relaxation of the magnetization in the first lanthanide complex of a 1,2,4-benzotriazinyl radical ${ }^{\dagger}$
}

\author{
Ian S. Morgan, ${ }^{a *}$ Akseli Mansikkamäki, ${ }^{a}$ Mathieu Rouzières,,${ }^{b, c}$ Rodolphe Clérac, ${ }^{b, c^{*}}$ and Heikki M. \\ Tuononen ${ }^{\mathrm{a}^{*}}$
}

The first lanthanide complex of a 1,2,4-benzotriazinyl radical (1), $\operatorname{Dy}(1)(\text { tbacac })_{3} \quad(2$, tbacac $=2,2,6,6$-tetramethyl-3,5-heptanedionato), was synthetised and found to have an antiferromagnetically ordered ground state with a metamagnetic phase diagram and a critical field of $0.91 \mathrm{~T}$ at $1.85 \mathrm{~K}$. The application of a small dc field revealed the single-molecule magnet behaviour of 2, illustrating the coexistence of long-range antiferromagnetic order and slow relaxation of the magnetization.

Over the last 25 years, single-molecule magnets $(\mathrm{SMMs})^{1}$ have attracted increasing attention from chemists and physicists due to their potential uses in spintronics ${ }^{2}$ and quantum computing. ${ }^{3}$ In these molecular materials, the slow dynamics of the magnetization, that is, their magnet-like behaviour, is thermally activated with an effective energy barrier, $\Delta_{\text {eff, }}$ that depends on the spin-state and the zero-field splitting of the molecular complex. ${ }^{4}$ In recent years, the synthesis of SMMs based on lanthanide ions has been an active topic in the field because of their inherent large uniaxial anisotropy and high spin, both of which increase the energy barrier $\Delta_{\text {eff }}{ }^{5}$ In fact, dysprosium and terbium ions possess such substantial anisotropy that the vast majority of lanthanide-based SMMs take use of these two metal ions.

Organic radical ligands have been shown to be an efficient strategy for enhancing magnetic interactions in lanthanidebased coordination complexes. ${ }^{6}$ While there are many lanthanide-based SMMs (and also single-chain magnets, SCMs) that contain radical ligands such as nitronyl nitroxides, ${ }^{7}$ semiquionates, ${ }^{8} \mathrm{~N}_{2}{ }^{3-},{ }^{9}$ azophenine, ${ }^{10}$ and thiazyls, ${ }^{11}$ only one

a. Department of Chemistry, Nanoscience Centre, University of Jyväskylä, P.O. Box 35, FI-40014, University of Jyväskylä, Finland. E-mail: ian.s.morgan@jyu.fi heikki.m.tuononen@jyu.fi

b. CNRS, CRPP, UPR 8641, F-33600 Pessac, France. E-mail: clerac@crpp-

bordeaux.cnrs.fr

c. Univ. Bordeaux, CRPP, UPR 8641, F-33600 Pessac, France.

† Electronic Supplementary Information (ESI) available: Full details of synthetic work and characterization by spectroscopic, magnetic, computational, and crystallographic methods. CCDC 1563199. For ESI and crystallographic data in CIF or other electronic format, see DOI: 10.1039/x0xx00000x

example of a lanthanide-verdazyl complex has been published to date; ${ }^{12}$ related lanthanide-bipyridine complexes, have, however, been extensively investigated. ${ }^{13}$ This is somewhat surprising considering the longevity of verdazyl radicals under ambient conditions and their propensity to coordinate to metals. In this context, we have recently reported a new related nitrogen-based coordinating radical, namely 1-phenyl-3-(pyrid2-yl)benzo[e] $[1,2,4]$ triazinyl (1), along with its $d$-block metal complexes. ${ }^{14}$ In this contribution, we extend the coordination chemistry of 1 to $f$-block metals and report the complex $\operatorname{Dy}(\mathbf{1})(\text { tbacac })_{3} \quad(\mathbf{2}, \quad$ tbacac $=2,2,6,6$-tetramethyl-3,5heptanedionato). Theoretical attempts to determine the exchange parameters and the origin of the long-range antiferromagnetic order in $\mathbf{2}$ are also described.

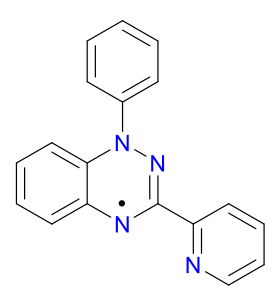

1

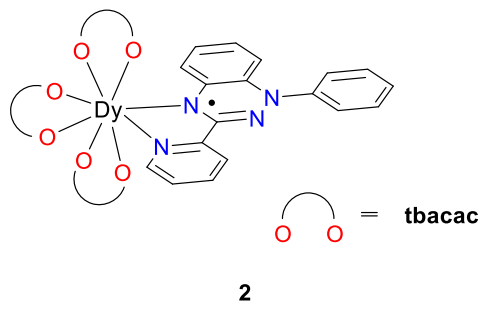

Complex $\mathbf{2}$ was synthesised by reacting radical $\mathbf{1}$ with anhydrous $\mathrm{Dy}(\mathrm{tbacac})_{3}$ in dichloromethane. Recrystallization from pentanes afforded air and moisture stable dark red single crystals of $\mathbf{2}$ that were suitable for X-ray diffraction and magnetic susceptibility measurements.

Compound $\mathbf{2}$ crystallizes in the monoclinic space group $P 2_{1} / n$. The asymmetric unit contains a single $\mathrm{Dy}(\mathbf{1})(\text { tbacac })_{3}$ complex in which the Dy"l' ion is coordinated by six oxygen and two nitrogen atoms that overall form a coordination environment that can be described as a distorted square antiprism or, alternatively, a distorted triangular dodecahedron (Fig. 1). Nearby complexes in adjacent asymmetric units interact 


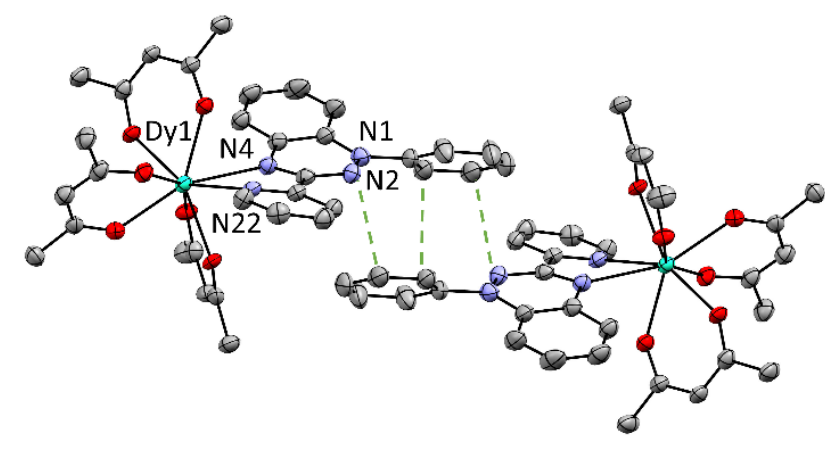

Fig. 1 ORTEP plot (50\% probability ellipsoids) of 2 . Two adjacent asymmetric units are shown to illustrate close contacts between neighbouring complexes. Methy groups and hydrogen atoms have been removed for clarity.

via close contacts between atoms on the $\mathrm{N}$-phenyl substituent of the radical ligand (Fig. 1). Pairwise interactions of this nature are commonly observed for many radical ligands and can result in strong antiferromagnetic coupling of their spins to the point where the radical-..radical unit becomes essentially diamagnetic. However, in case of complex 2, the closest C...C contacts (3.310(6) $\AA$ ) are only slightly shorter than the sum of van der Waals radii and involve atoms with little spin density.

The magnetic properties of $\mathbf{2}$ were measured at an applied dc field of 1000 Oe. The $\chi T$ product at $300 \mathrm{~K}$ is $14.4 \mathrm{~cm}^{3} \mathrm{~K} \mathrm{~mol}^{-1}$ (Fig. 2) which is in good agreement with the expected value for an isolated Dy'II ion $\left(S=5 / 2, L=5,{ }^{6} H_{15 / 2}, g=4 / 3 ; C=14.17 \mathrm{~cm}^{3}\right.$ $\left.\mathrm{K} \mathrm{mol}^{-1}\right)$ and one radical spin $\left(S=1 / 2, C=0.375 \mathrm{~cm}^{3} \mathrm{~K} \mathrm{~mol}^{-1}, g=\right.$ 2). It is difficult to determine the apparent (if any) magnetic contribution from the radical spin due to the intrinsic large paramagnetic contribution of the Dy"ll site. Upon lowering the temperature, the $\chi T$ product decreases to a minimum value of $1.1 \mathrm{~cm}^{3} \mathrm{~K} \mathrm{~mol}^{-1}$ at $1.8 \mathrm{~K}$. This thermal behaviour arises partially

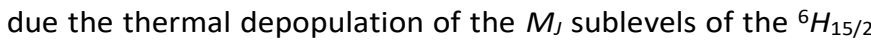
multiplet but also certainly from antiferromagnetic couplings in the material.

The field dependence of the magnetization below $1 \mathrm{~T}$ reveals a characteristic $S$-shape variation at a characteristic field $H_{\mathrm{C}}$ (defined as the maximum of the field derivative of the magnetization, $\mathrm{d} M / \mathrm{d} H$; Fig. $3 \mathrm{~b}$ ), confirming the presence of significant antiferromagnetic interactions. At higher fields, the

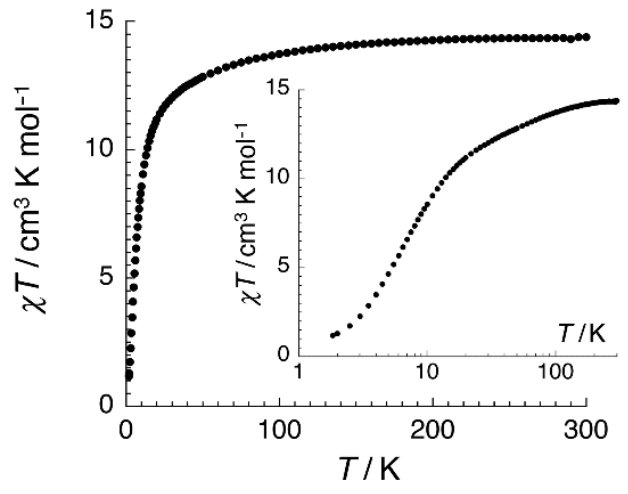

Fig. 2 Temperature dependence of the $\chi T$ product for $\mathbf{2}$ at 1000 Oe with $\chi$ defined as the molar magnetic susceptibility equal to $M / H$ per mole of 2 . Inset: $\chi T$ vs $T$ data shown in semi-logarithmic scale.
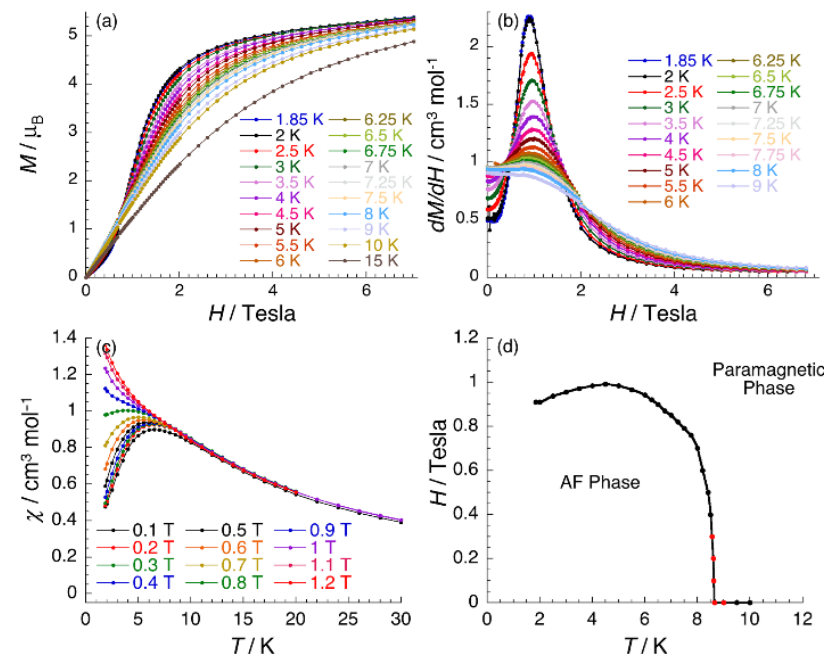

Fig. 3 Low temperature magnetic properties for 2: (a) $M$ vs $H$ and (b) $d M / d H$ vs $H$ plots with field sweep rates of $100-200$ Oe $\mathrm{min}^{-1}$; (c) $\chi$ vs T plots; and d) magnetic phase diagram $(H, T)$ constructed from $\chi$ vs $T$ (red) and $M$ vs $H$ (black) data. Solid lines are visual guides for the eye.

magnetization increases in slow and linear manner as expected for a Dy"I complex with significant magnetic anisotropy. Combining $M$ vs $H$ and $\chi$ vs $T$ data (Fig. 3a-c), the temperature dependence of $H_{\mathrm{C}}$ was followed from $H_{\mathrm{C}}(0)=0.91 \mathrm{~T}$ at $1.85 \mathrm{~K}$ to $0 \mathrm{~T}$ at $T_{\mathrm{N}}=8.6 \mathrm{~K}$, proving the occurrence of a magnetic phase transition line separating the three-dimensionally ordered antiferromagnetic ground state and a paramagnetic state (the resulting $(T, H)$ magnetic phase diagram is shown in Fig. $3 \mathrm{~d}$ ).

As found for most lanthanide systems that have very fast relaxation of the magnetization, no hysteresis effect on the $M$ vs $H$ data is observed above $1.85 \mathrm{~K}$ for $\mathbf{2}$. Thus, complex $\mathbf{2}$ was subjected to ac susceptibility measurements to examine possible slow dynamics of the magnetization. In zero dc field, ac measurements were performed down to $1.85 \mathrm{~K}$. Within the frequency range of 10-10000 Hz, no out-of-phase signal was observed. Therefore, the ac susceptibility was further studied under dc fields as this can have a double effect: (i) to lift the degeneracy of the energy levels and thus to minimize the quantum tunnelling of the magnetization in SMMs (usually at low dc field $)^{4}$ and (ii) to compensate for the antiferromagnetic interactions between magnetic objects revealing their intrinsic dynamic properties. This kind of decoupling effect has been observed in paramagnetic systems of $\mathrm{SMMs}^{11}$ but also in some SCMs displaying a three-dimensionally ordered antiferromagnetic ground state. ${ }^{15}$ In the former case, the observation of slow relaxation of the magnetization is possible at the vicinity of the antiferromagnetic-paramagnetic phase transition that for 2 occurs at $0.91 \mathrm{~T}$ and $1.85 \mathrm{~K}$ (Fig. 3d).

Under a dc field at $1.9 \mathrm{~K}$, a relaxation mode clearly appears with the slowest relaxation frequency of $1280 \mathrm{~Hz}$ observed at an optimum field of $0.85 \mathrm{~T}$, very close to the corresponding $H_{\mathrm{C}}$ found by static magnetic measurements (Fig. $3 \mathrm{~d}$ ). This result unambiguously confirms the decoupling effect of the magnetic field and the slow relaxation of the magnetization in $\mathbf{2}$. The ac susceptibility was studied under $0.85 \mathrm{~T}$ and below $15 \mathrm{~K}$ (Fig. 4) 

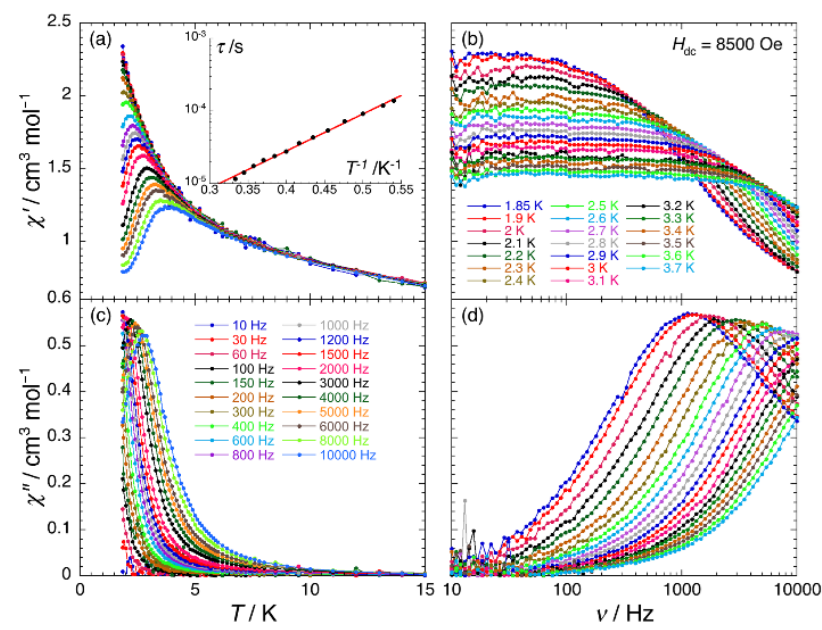

Fig. 4 Temperature (left) and frequency (right) dependence of the real ( $\chi^{\prime}$, top) and imaginary ( $\chi^{\prime \prime}$, bottom) parts of the ac susceptibility between 10 and $10000 \mathrm{~Hz}$ and guides for eye. Inset: $\tau$ vs $T^{-1}$ plot for 2 in 0.85 T dc field. Solid red line represents the best fit to the Arrhenius law.

to determine the temperature dependence of the relaxation time that appeared to be thermally activated (Fig. 4, inset). From this data, the effective energy barrier $\Delta_{\text {eff }} / k_{\mathrm{B}}$ can be estimated to be $11.8 \mathrm{~K}$ with an Arrhenius law pre-exponential factor of $\tau_{0}=2.5 \times 10^{-7} \mathrm{~s}$.

The lack of slow relaxation of the magnetization in the absence of an external field can be rationalized in terms of the crystal field around the Dy"II ion. It has been well established that the Dy"l' ion shows strong anisotropy in axial crystal fields ${ }^{16}$ whereas in 2, the crystal field has a distorted square antiprismatic geometry. In low-coordination environments, the quantum tunnelling of the magnetization is strong and slow relaxation of the magnetization can only be observed if the tunnelling process is suppressed by an external magnetic field, as is the case herein. To this extent, it is worth mentioning that slow relaxation of the magnetization has been observed for square antiprismatic Tb ${ }^{111}$ complexes. ${ }^{17}$ However, in $\mathbf{2}$, the immediate coordination environment around the metal ion contains oxygen atoms with formal negative charge and nitrogen atoms that are formally neutral, creating a charge distribution with symmetry considerably lower than that implied by the square antiprismatic geometry alone. It is therefore very unlikely that slow relaxation at zero-field would be observed if the Dy"II ion in $\mathbf{2}$ were to be replaced by Tb"', or any other lanthanide ion for that matter. In certain cases exchange interaction with the radical spin can also be used to suppress the quantum tunnelling of the magnetization, which is, however, not observed herein. The exchange coupling between the Dy'II ion and the radical leads to an overall nonKramers' system with an even number of electrons and, thus, to the loss of Kramers' degeneracy. This combined with the low symmetry of the crystal field in $\mathbf{2}$ most likely leads to faster relaxation of the magnetization rather than suppression of tunnelling.

The packing of $\mathbf{2}$ in the solid state indicated that $\operatorname{Dy}(\mathbf{1})(\text { tbacac })_{3}$ is structurally a mononuclear complex. The relative magnitude of the metal-radical magnetic coupling was estimated by scalar relativistic density functional theory (DFT) calculations conducted on a model system with Gd"I ion in place of Dy"l'. This allows the metal-radical coupling strength to be estimated in an identical ligand field as in complex $\mathbf{2}$ but without the need to consider the orbital contribution to the angular momentum. After rescaling to the $S=5 / 2$ spin moment of Dy'I', the calculated coupling $2 J_{\mathrm{D} y-\mathrm{R}}$ was found to be $-6.7 \mathrm{~cm}^{-1}$, suggesting that the metal-radical interaction is weak and antiferromagnetic. There are, however, also short radical...radical contacts that need to be considered. DFT calculations for two nearest-neighbour complexes of $\mathbf{2}$ (interacting via their $N$-phenyl substituents, Fig. 1) gave $2 J_{\mathrm{R}-\mathrm{R}}=$ $-15.8 \mathrm{~cm}^{-1}$, which shows that the radical $\cdots$ radical coupling is antiferromagnetic and too weak to support a complete magnetic dimerization at room temperature. Apart from these two magnetic couplings, no obvious exchange pathways that would induce the three-dimensional antiferromagnetic order in $\mathbf{2}$ could be identified from the crystal structure. Hence, at this stage, the stabilization of antiferromagnetic order is attributed to dipolar coupling between the Dy ${ }^{\prime \prime \prime}$ ions (the shortest throughspace distance between metal centres is $10.3358(4) \AA$ ) , assisted by weak radical-radical interactions. A more detailed theoretical analysis is currently underway.

\section{Conclusions}

Herein we have structurally, magnetically, and computationally characterised the compound $\mathrm{Dy}(\mathbf{1})\left(\right.$ tbacac) ${ }_{3}$ (2), the first lanthanide complex of a new coordinating 1,2,4-benzotriazinyl radical. Complex $\mathbf{2}$ is soluble into organic solvents and stable both in the solid state and in solution under ambient conditions, presumably owing to the favourable properties of radical 1 . Magnetic susceptibility data collected on $\mathbf{2}$ revealed an antiferromagnetically ordered ground state below $8.6 \mathrm{~K}$ with a metamagnetic phase diagram and a critical field of $0.91 \mathrm{~T}$ at 1.85 K. When a small magnetic field was applied and the intermolecular antiferromagnetic interactions compensated for, the single-molecule magnet behaviour of $\mathbf{2}$ could be observed, illustrating the coexistence of long-range antiferromagnetic order and slow relaxation of the magnetization. The successful characterization of $\mathbf{2}$ opens up a new possibility to use 1,2,4-benzotriazinyl radicals in lanthanide coordination chemistry. The versatility of the radical framework should allow for the design and synthesis of new paramagnetic ligands that are more adapted for coordination to rare earth metal ions as well as intelligent construction of novel mixedmetal systems that could be used as building blocks in the development of single-molecule magnets and, more generally, molecule-based magnets.

This work was supported by the Technology Industries of Finland Centennial Foundation, the Academy of Finland (projects 282499 and 289172), the University of Jyväskylä, the University of Bordeaux, the Région Aquitaine, the ANR, the CNRS, and the GdR MCM-2. We thank Lab. Tech. Elina Hautakangas for elemental analyses and Dr. Anssi Peuronen for crystallographic insight. 


\section{Conflicts of interest}

There are no conflicts to declare.

\section{Notes and references}

1 (a) R. Sessoli, D. Gatteschi, A. Caneschi, and M. A. Novak, Nature, 1993, 365, 141; (b) D. Gatteschi, R. Sessoli, and J. Villain, Molecular Nanomagnets, Oxford University Press, Oxford, UK, 2006.

2 L. Bogani and W. Wernsdorfer, Nat. Mater., 2008, 7, 179.

3 M. N. Leuenberger and D. Loss, Nature, 2001, 410, 789.

4 D. Gatteschi and R. Sessoli, Angew. Chem., Int. Ed., 2003, 42, 268.

5 (a) G. Poneti, K. Bernot, L. Bogani, A. Caneschi, R. Sessoli, W. Wernsdorfer, and D. A. Gatteschi, Chem. Commun. 2007 1807; (b) H. L. C. Feltham and S. Brooker, Coord. Chem. Rev. 2014, 276, 1; (c) R. A. Layfield, Organometallics, 2014, 33 1084; (c) D. N. Woodruff, R. E. P. Winpenny, and R. A. Layfield, Chem. Rev., 2013, 113, 5110.

6 S. Demir, I.-R. Jeon, J. R. Long, and T. D. Harris, Coord. Chem. Rev., 2015, 289-290, 149.

7 (a) F. Pointillart, K. Bernot, G. Poneti, and R. Sessoli, Inorg. Chem., 2012, 51, 12218; (b) P. Hu, M. Zhu, X. Mei, H. Tian, L. Li, and D. Liao, Dalton Trans., 2012, 41, 14651; (c) K. Bernot, F. Pointillart, P. Rosa, M. Etienne, R. Sessoli, and D. Gatteschi, Chem. Commun., 2010, 46, 6458; (d) H. Tian, R. Liu, X. Wang, P. Yang, Z. Li, L. Li, and D. Liao, Eur. J. Inorg. Chem., 2009, 4498.

8 (a) A. Dei, D. Gatteschi, C. A. Massa, L. A. Pardi, S. Poussereau, and L. Sorace, Chem. Eur. J., 2000, 6, 4580; (b) A. Caneschi, A. Dei, D. Gatteschi, S. Poussereau, and L. Sorace, Dalton Trans., 2004, 1048.

9 (a) J. D. Rinehart, M. Fang, W. J. Evans, and J. R. Long, Nat. Chem., 2011, 3, 538; (b) J. D. Rinehart, M. Fang, W. J. Evans, and J. R. Long, J. Am. Chem. Soc., 2011, 133, 14236; (c) S. Delmir, J. M. Zadrozny, M. Nippe, and J. R. Long, J. Am. Chem. Soc., 2012, 134, 18546; (d) S. Delmir, M. Nippe, M. I. Gonzalez, and J. R. Long, Chem. Sci., 2014, 5, 4701.

10 I.-R. Jeon, J. G. Park, D. J. Xiao, and T. D. Harris, J. Am. Chem. Soc., 2013, 135, 16845.

11 E. M. Fatila, M. Rouzières, M. C. Jennings, A. J. Lough, R. Clérac, and K. E. Preuss, J. Am. Chem. Soc., 2013, 135, 9596.

12 L. Norel, L. M. Chamoreau, Y. Journaux, O. Oms, G. Chastanet, and C. Train, Chem. Commun., 2009, 2381.

13 F. Ortu, J. Liu, M. Burton, J. M. Fowler, A. Formanuik, M.-E. Boulon, N. F. Chilton, and D. P. Mills, Inorg. Chem., 2017, 56, 2496.

14 (a) I. S. Morgan, A. Peuronen, M. M. Hänninen, R. W. Reed, R. Clérac, and H. M. Tuononen, Inorg. Chem., 2014, 53, 33; (b) I. S. Morgan, A. Mansikkamäki, G. A. Zissimou, P. A. Koutentis, M. Rouzières, R. Clérac, and H. M. Tuononen, Chem. Eur. J. 2015, 21, 15843.

15 (a) C. Coulon, R. Clérac, W. Wernsdorfer, T. Colin, and H. Miyasaka, Phys. Rev. Lett., 2009, 102, 167204; (b) H. Miyasaka, K. Takayama, A. Saitoh, S. Furukawa, M. Yamashita, and R. Clérac, Chem. Eur. J., 2010, 16, 3656; (c) I. Bhowmick, E. A. Hillard, P. Dechambenoit, C. Coulon, T. D. Harris, and R. Clérac, Chem. Commun., 2012, 48, 9717.

16 J. D. Rineheart and J. D. Long, Chem. Sci., 2011, 2, 2078.

17 N. Ishikawa, M. Sugita, T. Ishikawa, S. Koshihara, and Y. Kaizu, J. Am. Chem. Soc., 2003, 125, 8694. 